\title{
Oral Health Self-Care Products: Realities and Myths ${ }^{\S}$
}

\section{Sandra Liana Henz ${ }^{(a)}$ Ione Suslik Baron ${ }^{(b)}$ (organizers)}

(a) $\mathrm{PhD}$, Department of Preventive and Social Dentistry, School of Dentistry, Federal University of Rio Grande do Sul, Porto Alegre, RS, Brazil.

(b) MSc, School of Dentistry, Lutheran University of Brazil, Canoas, RS, Brazil.

§Summary of the Discussions held at the "Oral Health Self-Care Products: Realities and Myths" international symposium, sponsored by the Brazilian Association for Oral Health Promotion (ABOPREV), September 25-27, 2008, São Paulo, SP, Brazil.

Corresponding author:

Sandra Liana Henz

Departamento de Odontologia Preventiva e Social, Faculdade de Odontologia,

Universidade Federal do Rio Grande do Sul

R. Ramiro Barcelos, 2492, Santana

Porto Alegre - RS - Brazil

CEP: 90035-003

E-mail:slhenz@yahoo.com.br

\section{Dental biofilm and oral health diseases Can hormonal changes during puberty have an effect on the quality of biofilm?}

Ricardo Teles: It is difficult to know whether hormonal changes are causing behavioral changes, thus leading to gingivitis, or hormonal changes are directly affecting receptors in the gingiva, thus increasing sensitivity to the effects of plaque.

\section{Since an acid attack can be extremely severe, could erosive attacks kill cariogenic bacteria, thus preventing caries in people with erosion?}

Mark Wolff: Several individuals with an erosion process show very low caries rates, but this is not necessarily related to the elimination of cariogenic bacteria. These bacteria are aciduric and survive at very low $\mathrm{pH}$ conditions, so that situation would not lead to their elimination. But I agree with the fact that most of these patients have an extremely clean and smooth enamel surface and less caries. We have been taught to brush our teeth after meals; however, in the United States, people usually have an orange juice at breakfast - so, you drink a $\mathrm{pH} 3.5$ juice in the morning, and this will dissolve the enamel; then you brush your teeth and the mechanical action removes all the enamel that is softened or dissolved in the external layer.

\section{Regarding biofilm thickness associated with cariogenicity and periodontopathogenicity, are there any associations and what is their clinical relevance?}

Ricardo Teles: Regarding biofilm thickness in relation to periodontal diseases, it is important to make a distinction between gingivitis and periodontitis, that is, biofilm thickness and plaque accumulation have a well-established and direct relationship with gingivitis, which is well documented in the literature. Regarding periodontal disease, this association may be found in patients with moderate and more clinically localized periodontitis. When we refer to severe disease, with possible tooth loss, this relationship is less evident. A classic study, the study by Löe et al. with Sri Lankan laborers at tea plantations with poor oral hygiene, demonstrated that, despite the absence of plaque control, a reasonable number of individuals (11\%), who had been exposed to dental plaque for decades, did not present loss of attachment. In that study, $80 \%$ of individuals showed some moderate attachment loss, and only a small number of people $(8 \%)$ developed more advanced attachment loss. This takes us back to the original question of what is the relevance of this thick- 
ness, this excess, and this volume of bacterial burden for the onset of more severe periodontal disease. Yet, this does not rule out microbial participation in these diseases; actually, it highlights the fact that periodontal disease is a multifactorial disease which requires several conditions to be present in order to appear. These observations suggest how efficiently the immune system can deal with this challenge. In other words, almost everyone will show accumulation of bacteria throughout life, but not everyone will actually develop periodontal disease, particularly severe forms of the disease. These findings also indicate that the composition of this biofilm needs to have a certain specificity in order to lead to the development of periodontal disease. These observations were also useful to make a distinction between the two disease processes. Gingivitis would be considered as an essential process to establish the conditions necessary for the development of periodontitis, but without an actual continuum from gingivitis to periodontitis. That is to say, periodontitis is not necessarily an obvious, essential and direct consequence of gingivitis. It is not only a matter of length of exposure to gingivitis, especially if we refer to severe periodontitis. Over the last years, there has been a growing trend toward a recall of this interpretation that gingivitis inevitably results in periodontitis. This notion that gingival inflammation is necessary to set conditions for the development of periodontitis is certainly important; however, what triggered the recall of periodontitis as a subsequent development of gingivitis was the association between periodontal and systemic diseases. All correlations found between systemic and periodontal diseases are questionable and, when a stronger association was found, it was related to gingivitis. Plaque thickness surely has a direct relationship with gingivitis, with increased risk of attachment loss and development of primarily moderate and localized periodontitis in the areas where an excessive biofilm accumulation is observed. The relationship between plaque thickness and severe periodontal disease, however, is weaker and less direct.

Mark Wolff: Dental plaque thickness has little to do with its cariogenicity issue; thickness is more closely related to the number of bacterial layers. There is plenty of evidence that the thicker the plaque becomes, the greater the conditions for anaerobiosis are; therefore, this is much more connected to gingivitis [rather than periodontitis] development. The question about permitting interruption of plaque maturation arises exactly because we can clearly perceive the effect of the relationship between plaque removal - or maintenance of this less mature plaque - and gingivitis reduction, which cannot be so clearly observed in relation to caries. The longer plaque remains on the tooth, the greater the chances that acidogenic bacteria will be present and produce acid. It does not mean that a thick biofilm will not become cariogenic and is unimportant to caries, but what I mean is that even a very thin biofilm may already be cariogenic. There is a series of studies which attempted to observe plaque accumulation using the plaque index by Löe \& Silness. None of these studies managed to demonstrate higher caries rates correlated with higher loads of biofilm thickness.

\section{Plaque mechanical disorganization is not effective in the reduction of caries; it is closely related to gingivitis, not to caries.}

Jaime Cury: Plaque thickness plays an important role, even in dental caries. Data from the literature show that very clearly from a biofilm $\mathrm{pH}$ fall standpoint, and I agree that the type of biofilm formed is important. You need a biofilm maturation period of approximately 5 days for an enamel critic $\mathrm{pH}$ fall to occur from a caries standpoint, but what is more important here, what we have been discussing about, is the type of dietary carbohydrate in relation to the formation of this biofilm. You have a thick biofilm in which antimicrobial agents do not penetrate, but sugar does. Then, what happens? You will see the difference in the maturation of these biofilms, if they are formed in the presence of sugar or not. Thus, when biofilm is formed in the presence of sucrose, you can observe pores that allow a rapid diffusion of sugar into the biofilm, with a markedly critic $\mathrm{pH}$ fall next to the tooth. Then, what happens? The antimicrobial agent does not penetrate, killing bacteria on the surface only, but sugar does penetrate. Therefore, biofilm disorganization also 
plays an important role from a caries standpoint.

Previous studies have also discussed the importance of biofilm in terms of first mechanism of $\mathrm{pH}$ fall in relation to dental caries. Studies show the effect of biofilm thickness on enamel remineralization: when biofilm was thick, enamel remineralization would take longer than with a less thick biofilm. This assertion indicates that there is an association between maturity and biofilm thickness. I am not discussing maturity and biofilm thickness, the effect on mineral loss or the effect resulting from an attempt at replacing the amount of mineral loss. So, this takes us or draws attention to the importance of biofilm disorganization, or even of the disorganization itself, as in the discussion on antimicrobial effects.

Mark Wolff: And none of these studies managed to demonstrate higher caries rates correlated with higher loads of biofilm thickness. In addition, we need to remember that thicker biofilms have a much more complex ecology, containing calcium, fluoride and alkaline formations.

Audience: It seems that part of this discussion lies in how thick this critical mass is or should be in order to develop, or not, cariogenic plaque. In fact, for a long time no obvious relationship between population-based oral hygiene parameters and caries rates could be found. But I remember that [discussion] and how much that has bothered me, because we already talked about all these concepts of microbial etiology of caries at that time, but they could never convince me, as the arguments sounded almost like a statement against the need for a standard oral hygiene.

Subsequent studies demonstrated that plaque control can actually reduce caries. The question lies in the level of plaque control required in order to have an anticariogenic effect, and the plaque control observed in the population may not be at a level that, in the absence of fluoride, could control cariogenic processes. It goes without saying that the greater the amount of plaque, the stronger the cariogenic effect, which makes it more difficult to control this process.

Mark Wolff: I am not saying that people should stop brushing and flossing. What I mean is that, if people make use of brushing and flossing as the only approach, this will not be enough to interrupt or reduce caries. As a dentist, I still instruct patients on brushing and flossing, but for several other reasons, including reasons related to systemic diseases and all those other aspects well-known to us. I want to state that the importance of brushing and flossing within the biofilm issue is not crucial and cannot resolve a caries problem on its own.

Marisa Maltz: We agree that caries control based exclusively on biofilm control is extremely difficult; however, we should remember that caries control is closely associated with biofilm periodic control. We should take into account, in addition to biofilm thickness, the frequency of biofilm removal in the control of dental caries. There is evidence that, for example, periodic removal of biofilm once a week does not cause clinically visible mineral loss within a 5 -week experimental period. On the other hand, if the biofilm remains disorganized and on dental surface for over a week, clinical signs of mineral loss can already be observed. Thus, periodic mechanical removal of biofilm is important and should be taken into consideration in the treatment of our patients.

\section{Since supragingival plaque has a certain influence on subgingival plaque, does biofilm thickness have to do with a greater susceptibility to periodontal disease or not?}

Ricardo Teles: Evidence shows an extremely direct relationship with gingivitis. Gingivitis is a risk factor for loss of attachment and progression to periodontal disease, evidence from the literature supports this in a clear and direct manner. The question is: what is the impact of this on more severe and aggressive forms of periodontal disease that lead to dental loss? In these cases, evidence is scant, correlation less direct. The process is not as simple as: increased thickness leads to increased anaerobiosis, which leads to increased inflammation, which, in turn, will unavoidably lead to loss of attachment. There are, literally, "attachment-lossresistant people", who, although exposed to thick plaque and with gingivitis, go through life without developing attachment loss. Little is known about the mechanisms that lead to this "resistance". There 
are several theories to explain this observation: these individuals would have an immune response more suitable to deal with the microbial challenge, or their biofilm would show a distinct characteristic that would prevent the development of the disease; however, knowledge on the details of this mechanism is scarce. Let us bring in localized juvenile periodontitis to remind us that there are periodontal disease conditions in which no biofilm thickness is needed for the development of severe loss of attachment. Refractory periodontitis is another good example. These conditions are rare, with an incidence of $1 \%$ of periodontitis cases, and it takes years to appropriately diagnose them. Studying this type of condition is quite difficult, a long-term largescale randomized study of refractory periodontitis patients would be almost impossible to be accomplished. However, if you have already treated a case of refractory periodontitis, you know that this condition can also be characterized by very little plaque. This does not mean that refractory periodontitis does not have a microbial etiology; strong evidence of this remark lies in the actual response to appropriate antimicrobial therapy. These conditions clearly show that there is no need for plaque thickness in order to develop loss of attachment. It is worth mentioning that plaque control is yet the best way to prevent periodontal disease in most individuals, but there are several conditions in which these observations do not work in such a direct and absolute manner, and understanding why these conditions behave in such a different way is an ongoing challenge for periodontitis.

\section{Dental biofilm and chemical agents What is the cost-benefit relationship of using antimicrobials to assist in the control of gingivitis?}

Ricardo Teles: It is difficult to justify on costbenefit grounds a population-based approach. If Triclosan was made more available and as cheap and easy to be mixed in the toothpaste as fluoride is, I believe that we would already have enough data to justify and include Triclosan in the composition of the toothpaste that the Brazilian Ministry of
Health will distribute to the population. However, if this entailed reducing the budget for the hiring of dental hygienists who are instructing people on how to correctly brush and floss, we would rather hire the hygienists to teach brushing and flossing, which should take place in the school setting. There are two opportunities to educate people: at home (in the family) or at school.

\section{About the use of chlorhexidine}

Ricardo Teles: For an immediate and short-term plaque control, for example, conducted postoperatively, chlorhexidine remains as first choice. $\mathrm{Ny}$ man [et al.] demonstrated that in patients without plaque control after surgery, plaque develops considerably more in comparison with those who are on appropriate postoperative plaque control. They tried to counterbalance this lack of plaque control by using chemical control: chlorhexidine was used for 6 weeks in patients who had not received careful orientation regarding postoperative mechanical plaque control. The effect was lower than that of the group on appropriate mechanical control. Disease progression was not observed in this study as it was in a study in which patients were not on any maintenance therapy, but mechanical control or an appropriate maintenance resulted in healing rates significantly higher than those obtained with chemical control alone. These results may be explained by an interference of biofilm with chlorhexidine's antimicrobial effect, that is, a well-performed mechanical plaque control can yield better results. Chlorhexidine can be complementary to or replace mechanical plaque control, but, after two, three or four weeks, extrinsic stains will appear.

Cassiano Rösing: In self-care situations, there are two things that cannot be left aside. First, the nature of biofilms: that they need to be mechanically disrupted so that they can be, somehow, modified. Then, this leads us to the need for some removal or mechanical disruption and, extending the topic beyond mechanical control into chemical agents sold as self-care agents, the thicker the biofilm is, the less effective the agent is, even those agents in a toothpaste; therefore, even if we use a potent agent such as chlorhexidine, if the mouth is full of plaque, 
chlorhexidine will not work out. In a study published in 2007 in the Journal of Periodontology, we tried to use chlorhexidine both in plaque and gingivitis development in areas with 4-day plaque accumulation, and chlorhexidine did not work out; it actually induced tooth-stain and calculus formation. Therefore, chlorhexidine, as well as other chemical agents, cannot be prescribed over the phone.

\section{Dental bleaching What could you tell us regarding safety in the use of bleaching products by the general population?}

Flávio Demarco: Regarding safety, the gold standard would be $10 \%$ carbamide peroxide when used with mouthguards and for up to 4 weeks. Is there any adverse effect? There are studies reporting up to $30 \%$ tooth sensitivity or gingival irritation, which could potentially become a problem. However, products are not usually used as recommended, so you have an overtreatment. As in all cases of overtreatment, you have problems then. Sensitivity is temporary and can be interrupted by suspending the bleaching agent for 24 hours or by applying an agent that stops sensitivity.

Someone has asked me about long-term continuous use. There is a study by Bruce Matis, from Indiana University, published in Operative Dentistry, on bleaching teeth with tetracycline staining, in which patients underwent a 6-month bleaching treatment, at $10 \%$, and then were followed up for 5 years. That study was not carried out in the United States, but in China. What have they observed? Even with a 6month application, the adverse effect reported was sensitivity, and patients were instructed to stop the treatment, use fluoride, etc. After 5 years, the authors observed that the bleaching effect remained.

The main concern is regarding carcinogenesis though. I have cited a study by Treadway, a group from England which has many publications on this topic, and with respect to safety they concluded that carbamide peroxide had no effects in relation to carcinogenesis. Regarding carcinogenesis, references are associated with hydrogen peroxide, but in a study in which the authors assessed the potential to develop malignant lesions in rats, a huge amount of hydrogen peroxide was used, incompatible with the amount clinically used.

Toxicity is related to the amount of product used, and anything in excess is likely to be harmful. As I pondered at the end of my presentation, regarding a carcinogenic potential, hydrogen peroxide would play an adjuvant role when used in combination with tobacco, alcohol and taken in large amounts. According to the French Academy of Dentistry, the ideal amount to be used is $10 \mathrm{mg} / \mathrm{Kg}$. What is recommended then? To use low doses, preferably carbamide peroxide, and not for a very long period, thus being the safest material to be employed.

\section{Dental erosion}

What is the prevalence of dental erosion in children, adolescents and adults? Is there any evidence of an increase in this incidence? And in the diagnosis of erosion, which are the gingival characteristics associated with erosion?

Mônica Serra: According to Lussi, Jaeggi in 2008, a study published in 2003 by Dugmore, Rock, with 1,308 children in the United Kingdom, at a first assessment a prevalence of $4.9 \%$ was observed at 12 years of age.

Is there any evidence about this increase? Yes, there is, because within two years of study, this prevalence increased from almost 5 to $13.1 \%$ in 2 years. Regarding prevalence in adults, it is higher in adults aged 52-56 years than in adults aged 32-36 years, according to data from that study. Regarding evidence of soft toothbrush and erosion, I would like to clarify the nomenclature used for different types of wear, such as abrasion, abfraction and erosion.

In biotribology, "abrasion" is used for what dentists call "attrition". When antagonist teeth rub against each other, two-body abrasion occurs. Brushing without toothpaste would also be a twobody abrasion. When people chew their food, or when they brush their teeth with toothpaste, this would be considered a three-body abrasion. What many dentists call attrition, tribologists call twobody abrasion. And what dentists call abrasion is, more specifically, a three-body abrasion. In the case of "erosion", the most suitable term in biotribol- 
ogy is corrosion, since wear is caused by chemical action. What some people call abfraction would be the sum of several types of wear which lead to surface fatigue. Then, in the case of soft and hard toothbrushes, there is plenty of evidence regarding abrasive wear and, for that, a two-body test has to be present, only toothbrush and tooth. There is evidence that a harder toothbrush may wear the tooth structure to a greater extent. I am not aware of data on whether the association erosion/abrasion would be stronger, but, by analogy, I would believe so.

Mark Wolff: About the distinction between erosion and abrasion processes, basically, these processes may be or not distinct, but as for the way they have occurred in nature, they are likely to be associated, that is, the erosion process softens the enamel and then the mechanical process is introduced, aggravating that erosion process via abrasion.

\section{What would abfraction be?}

Cassiano Rösing: In fact, abfraction is a term from the Latin "ab" meaning away, and "fractio" meaning fracture. This term has been used to designate lesions that would occur at the enamel, dentin and cementum junction, which theoretically would have different stimulus-response coefficients, and fracture could occur under different occlusal forces. However, regarding this issue, to date, no one could demonstrate in vivo that lesions from occlusion may occur in the cervical area, there is no study in vivo showing this.

Mônica Serra: In biotribology we call cervical lesions fatigue lesions, and not abfraction.

\section{Is the position regarding abfraction a matter of nomenclature?}

\section{Cassiano Rösing and Mônica Serra: No.}

Audience: I observe that a higher incidence occurs in the premolars, which are teeth that, according to their occlusal position, are midway between posterior and anterior teeth; then, they would be more affected by expelling movements, as well as by toothbrushing.

Audience: I believe it's very clear that the cavity form is quite different from that of a lesion due to abrasion or from an abfraction. And, as mentioned by Mônica Serra, in engineering, in materials, we use the term fatigue meaning the submission of a body to deformation, and this process will tire the structure out, which will then suffer fatigue.

Audience: To which forces are our teeth exposed in the mouth, if not to occlusal forces and mastication? Then, why is not abfraction related to occlusal forces? And after you chisel these cervical prisms, which are very slim, abrasion by toothbrushing and erosion by dietary acids intensify this lesion, this is how I understand it. So, I would like to hear your opinion about it.

Cassiano Rösing: I examined all possible evidence from the literature that could establish a causal relationship, and none of these studies managed to establish a causal relationship. I do not mean that such relationship does not exist, but that, to date, it is yet to be confirmed.

Mônica Serra: There is an interesting study by Pegoraro [et al.], from Bauru [state of São Paulo, southeastern Brazil], in which he tried to relate patients; models were molded and mounted on an articulator, and the author observed that in cases with eccentric contact the teeth developed a small step in the cervical area (patients were followed up longitudinally).

Cassiano Rösing: The major problem of that study, and it is a rather interesting study, is that follow-up is carried out without covering the lesion area, and then there is no blinding to the lesion area, which makes it easier than by subjective attempts, but that is the only study available on the subject. There is a previous one in which the author used dry teeth, hitting on the tooth until it broke; of course, it would obviously break, but we tend to forget about the periodontal ligament, which takes after our same fault when talking about occlusion and periodontal disease in such a fanatic way. Today, we know that the relationship between occlusion and periodontal disease is minimal, if existing.

Mônica Serra: Then, there is this study with the criticism mentioned by Cassiano Rösing and there are studies using finite element method in biotribology which report that, when occlusal load is present, resulting forces will occur. And especially in the premolars, the strongest resulting force affects 
the cervical area. My opinion is that it can actually happen, but the presence of other related factors is essential and, because of that, I choose to call it fatigue, because people understand abfraction as if occlusal load alone could cause that lesion. In any type of lesion caused by wear there is fatigue. However, when the challenge is mainly "erosive" (corrosive), the resulting lesion is large, shallow, rounded. When occlusal load actions are also present, the lesion has a different shape; it is wedge-shaped, with clearcut angles. But, irrespective of the type of wear, all noncarious cervical lesions are, in my point of view, caused by fatigue.

Audience: How big are these lesions, and are they either self-limiting or progressive?

Ricardo Teles: Not really, there are some selflimiting lesions. Occlusal trauma - it is not an easy task to identify the effect of an occlusal trauma on periodontal disease. I believe that it occasionally happens, due to its self-limiting nature, because every time you overload a tooth, this tooth wears away, or moves, or causes the ligament to disperse. And when some of these adjustments occur, it is over. If you overload an area "more susceptible to fracture", so to speak, after the fracture occurs and the enamel is brushed off, the problem ends there.

Mark Wolff: First of all, in the United States there is an ongoing discussion on whether it is an abfraction or not, if abfraction actually exists and is really important, and whether there is a real need for treatment of these lesions. Think with me, what is the use of removing this functional or hyperfunctional tooth if it will not supra-erupt again and resume that previous position and for, at least, a quite long period of time? We know that filling materials do not solve the problem; however much we use this material with the aim of strengthening the tooth, we may see a leakage in the restoration and the development of a new lesion between the tooth surface and the restoration.

Hamilton Bellini: I would like to give my opinion as a clinician, and a clinician with a particular situation because I have clients who have been followed up for over 40 years, some of them at 92 years of age, for example, showing a tooth fracture which was... I am not sure how to call it, but [the tooth] had this cervical lesion and, in fact, I had more than one case. I have followed up, for a long time, two clients who are, now, over 90 years old. Both cases are associated with bruxism, and my clinical impression is that as these people age, bruxism enhances, and as this bruxism enhances, lesions seem to get worse anyhow, and, as Mark Wolff has mentioned, sometimes we perform a restoration in order to be able to clean it, because cleaning is difficult in such situations, but this does not prevent the tooth from fracturing at a certain point. But there is no doubt that we need to learn more about this subject. I believe that knowledge will develop over the next years, because more and more cases are arising since people are living longer; then people can be followed up for a longer period of time, and we can only learn from all that. So, I agree with you because I believe that there is an occlusal component which interferes with these cervical lesions in some cases. How many? I do not know!

What we should try to do, should I try to isolate that tooth from premature contacts? I try it, because premature tooth contact will undoubtedly result in some type of force on the tooth, a lateral force for which the tooth is not suitably prepared, right? And about bruxism, this is dynamic, because you adjust it one day and after two or three years you can observe parafunctional wear, which is not functional wear, and new contacts will occur; so, we definitely have to learn more about it, and we have the chance to do it as we have been having more and more clients in an older age group.

Audience: Yes, well, your dentin is exposed, but an exposed dentin is something that will ultimately happen with aging in what, 90 or $100 \%$ of the population? Probably in a $100 \%$. Exposed dentin is something that will happen to all of us, unless you do not have it. I currently have exposed dentin and do not think that my exposed dentin needs treatment.

\section{Dentifrice, caries and periodontal disease What is the indication for the use of dentifrice containing 5,000 ppm?}

Jaime Cury: For the dentifrice containing 
$5,000 \mathrm{ppm}$, the only indication, based on clinical studies, would be carious dentin; this is the only evidence for which there is an indication. In three clinical studies this dentifrice containing a high fluoride concentration, 5,000 ppm F, was more efficient than a conventional dentifrice $(1,100 \mathrm{ppm} F)$ in remineralizing or rehardening; lesions were not measured inside, though; the authors only measured clinical changes that occurred in the demineralized dentin; and it became harder, showing better results than with the dentifrice containing 1,100 ppm. This dentifrice was introduced in Brazil, but it could not be marketed in supermarkets because, according to Brazilian legislation, which follows Mercosul legislation, dentifrice containing fluoride concentration over $1,500 \mathrm{ppm}$ cannot be freely marketed, being available to consumers at pharmacies only.

\section{Colgate Total $^{\circledR}$ Triclosan should or could be used in children aged 12 years or older. Are there any contraindications to its use in under-12-year-old children?}

Ricardo Teles: In the instructions of Colgate To$\mathrm{tal}^{\circledast}$, the manufacturer recommends its use in over6 -year-old children. This recommendation may be due to risk of toxicity associated with fluoride rather than with Triclosan. So, our concerns are: can children use small amounts? Will children swallow that toothpaste? Can children spit most of that toothpaste? If we have such a control over this child, what I believe we only have over our own children, I do not see any problems in using it even before the age of 6 . The need for it is also questionable, but, once again, the manufacturer recommends its use only in children over 6 years of age.

Audience: I think that there is one thing we, dentists, should try to spread out: the need for a dramatic reduction in the amount of dentifrice used in our country. At any age, we use too much toothpaste, and I believe that this reduction should be recommended by every single dentist, regardless of containing or not Triclosan, or any other thing of that kind.

In a slide by Jaime Cury, he compared what he used to write 50 years ago and now, and he was already on the right track back then. First, he used to write: "brush your teeth and use fluoride toothpaste". Now he has the same recommendation: "brush your teeth, use little toothpaste". I think that this is what we have to learn, that we tend to exaggerate the use of toothpaste in our country, around $600 \mathrm{~g}$ per capita, and this represents a problem at any age, and we, dentists, must spread the news.

Mark Wolff: I would like to comment on that. All this comes from an agreement at the industry level, it was not regulated by FDA. There is an agreement that all manufactures in the United States should recommend the use of any toothpaste only in children over 6 years of age, neither because of Triclosan, nor because of antiplaque agents, but because of fluoride.

Audience: Sometimes I get concerned about the use of these antimicrobial agents in children, especially because long-term studies attempting to inhibit mother-child transmission or to change the microbiota of these individuals in order to demonstrate significant and important effects over time are yet to be conducted. Therefore, there are no longterm studies showing possible changes that could have occurred in the resident microflora.

Audience: I mean that there is a possibility, as you are in a stage of acquisition or development of your oral microbiota, which will become your indigenous microbiota and will ultimately be with you throughout life, that starting on antimicrobials at such an early age may have an effect on the development of this microbiota; an effect that we do not understand or know yet. And, in my opinion, this should be taken into account when considering the introduction of Triclosan at such an early age.

Jaime Cury: I am not aware of this recommendation by the manufacturers that only children over 6 years of age could use the toothpaste, because this recommendation does not correspond to our Brazilian legislation, and it sounds strange to be mentioned only in this toothpaste's instructions. So, in my opinion, if in Brazil this is mentioned only in this toothpaste's instructions, it is related to Triclosan, because in all other Brazilian toothpastes containing fluoride we do not find such a recommendation.

Mark Wolff: In fact, this is an attempt by the industry to escape from FDA regulatory issues, 
then, before FDA interference, because this generates a major problem situation, they make some agreements and reduce it; that is what happened to the toothpaste case. For example, in toothpastes containing fluoride concentrations of 1,400 or $1,500 \mathrm{ppm}$, a recommendation that a dentist should be consulted about how to use the product was provided on the toothpaste's carton in order to facilitate the regulatory situation.

Marisa Maltz: I consider this discussion very important mainly regarding the indication of fluoride dentifrice for under-3-year-old children. The use of fluoride dentifrice should be indicated for children, but controlling the amount to be applied on the toothbrush to avoid the occurrence of dental fluorosis.

Jaime Cury: First of all, there is no evidence supporting that the use of non-fluoride toothpaste to brush the teeth reduces caries. Secondly, this discussion about using non-fluoride toothpaste until the child turns 3 years old and assuring parents that the child will not develop fluorosis, I suggest that you do not suggest this, you know why? I was in Ponta Grossa [state of Paraná, southern Brazil] recently and we had already warned people before, and one of my colleagues attending the course said: "you see, my children have never eaten fluoride toothpaste, I have had them under strict control, and both of them developed fluorosis." I asked: "where do you live?" "Ah, here where there is fluoride in water." Thus, if a city supplies fluoridated water, this represents a higher risk factor than the use of dentifrice, which is estimated to contribute to $10-15 \%$ of the total prevalence of the resulting fluorosis. Evidence of this can be found in a survey on fluorosis carried out in Manaus [state of Amazonas, northern Brazil], where fluoride water is not supplied; there was a reduction in caries, and $65 \%$ of children in Manaus apply toothpaste to the whole bristle extension. What is the prevalence of fluorosis in Manaus, where fluoride water is not supplied? Fluorosis prevalence is approximately $12 \%$, and this value is in accordance with the increase in fluorosis that has been observed in Brazil since 1990, when fluoride dentifrice started to have an impact on the population of our country. I do not mean that fluoride dentifrice is not related to fluorosis, but I ask: what is its impact on the prevalence of fluorosis in Brazil? This issue has to be discussed.

Why do we say "do not use fluoride toothpaste in under-3-year-old children" instead of saying "use a small amount, after you turn 3 years old you can eat as much toothpaste as you want.” The educational process regarding the use of fluoride dentifrice in children needs to be revisited, taking into account the available evidence in terms of benefits (caries reduction) and risks (fluorosis) about the use of toothpaste in children. The same applies to the use of dentifrice containing low fluoride concentration, $500 \mathrm{ppm}$ F. If children keep on eating this toothpaste as much as they used to eat the $1,000 \mathrm{ppm}$ one, how can you assure that they will not develop fluorosis? Based on existing evidence, if a small amount of dentifrice, such as 1,000-1,100 ppm F, was used, this would better contemplate the binomial benefits/ risks in the use of fluoride than other alternatives that have been suggested.

Marisa Maltz: It is important to state that, when we carried out the survey on caries and fluorosis in Manaus together with Augusta Rebelo, we employed the same methodology of the study carried out in Porto Alegre [state of Rio Grande do Sul, southern Brazil], which is the master's thesis of Berenice B. Silva, and we found in Porto Alegre, a city with available fluoride water and fluoride dentifrice, a fluorosis prevalence around 50\%, significantly lower than that found in Manaus, where fluoride dentifrice is available but fluoride water is not supplied, meeting exactly what Jaime Cury has been discussing.

\section{What is the severity of this fluorosis you have mentioned, of this almost $50 \%$ increase? Are you talking about lesion, enamel alterations or staining?}

Marisa Maltz: We are talking about enamel staining, white spot lesion and not cavity. It is important to highlight the clinical significance of fluorosis. While conducting the study by Berenice in Porto Alegre, we observed a high prevalence of fluorosis. In 300 questionnaires, we included a question on whether people had any complaint concerning their teeth. Our goal was to assess whether that 
fluorosis was extremely weak, ITF level 1 and 2, which can only be seen in dry teeth without biofilm, or whether that fluorosis really had a social impact. We observed that if people complained about a dental position, people complained about anything, but in terms of staining there was no complaint.

Jaime Cury: I would like to add that no one is saying that dentifrice is not a risk factor for fluorosis. Dentifrice is a risk factor for fluorosis. The meaning of this statement needs to be discussed. I would like only to report an article of ours recently published in the Revista de Saúde Pública. I have been in contact with what is going on in Brazil for a long time, including the control of fluoride in water. So, we analyzed two cities here in [the state of] São Paulo, one of them has a perfect heterocontrol of fluoride in water, maintaining an excellent concentration of $0.7 \mathrm{ppm} F$ for over 10 years. The other city has oscillating water fluoridation, one day it is 0.3 , on the other day it is $1.3 \mathrm{ppm} \mathrm{F}$, with fluoride concentration varying in water. In the city with excellent fluoridation, fluorosis prevalence in children was $80 \%$, but in the city with irregular fluoridation fluorosis was twice as low. Another question arises from this then: what is the clinical significance of this fluorosis? Children were asked about satisfaction with their teeth: yes, no, why? None of the children reported being unsatisfied because of fluorosis. The prevalence of fluorosis was high, but most children showed fluorosis degree around 1, which does not result in lack of satisfaction with the appearance of teeth.

\section{What is the indication for $0.05 \%$ mouthrinses and is there any relationship of this concentration with fluorosis?}

Jaime Cury: Mouthrinsing is considered today as a complementary method to fluoride, in patients who already use fluoride dentifrice, and has a very particular or individual indication, although mouthrinses are considered a best-selling product in the market. I think it was Flávio Demarco who said that, I am not sure now, it was Cassiano Rösing, he said that, according to the latest national data, mouthrinses are best-selling products, reaching the highest sales rates over the last years. Consumers do not buy mouthrinses because of fluoride to control caries, but to have fresher breath. Currently, fluoride mouthrinsing, with the decline observed in caries, is only recommended when there is a high risk of caries or a high caries activity, on both a population and individual basis. Several examples could be considered indication data.

Marisa Maltz: We have to remember that in a $0.05 \%$ fluoride solution, we have a fluoride concentration of around $250 \mathrm{ppm}$, which is a low concentration. We recommend toothbrushing first, followed by mouthrinsing, then, in fact, we are not increasing the frequency because we are performing toothbrushing and, right after, mouthrinsing. We presented at the ORCA Congress a study in situ comparing a group using fluoride dentifrice twice a day, a second group using fluoride dentifrice twice a day, but the second brushing was followed by mouthrinsing, according to standard recommendations, and a third group with toothbrushing frequency increased to three times a day. The group with increased toothbrushing frequency had a result much better than that of the group on mouthrinsing following toothbrushing with fluoride dentifrice. Pointing out that, in relation to mouthrinsing, we are using an extremely low concentration. We currently know that the mechanism of action is topical fluoride. In patients with high cariogenic activity, I really want to enhance the fluoride effect, then it is interesting to increase frequency and also think of mouthrinsing with a $0.2 \%$ fluoride concentration.

\section{Symposium conclusions}

Jaime Cury: I would like to talk about the importance of ABOPREV in these discussions. I think that Nilce Tomita stated it very clearly yesterday that ABOPREV has reliability, credibility and identity in these discussions, and that we have to give these discussions a formal status. We should revisit and rediscuss remineralization, and the discussion on remineralization was confused with the discussion carried out in the past on the treatment of white spots. So, somehow I tried to demystify this part so that people could move on from there, and I think that this is not what occurs in this forum, trying to solve the problem of dental caries by simply treating 
the signs or symptoms of the disease. ABOPREV was responsible for all paradigm and concept shifts that have occurred, and within these concepts what I notice in this discussion on treatment or remineralization is rather a strategy from a perspective that it is easier to show effects; it is very easy, not difficult, to show effects, to gain some mineral in the enamel or dentin. In my opinion, the most important aspect is to discuss relevance within a health care setting/ context. Within a health context, it is important to interfere with the health-disease process, and then a white spot can relapse or not. What matters is maintaining patients healthy or without disease; so, the relevance of this approach represents, in my opinion, the great discussion, although there is evidence that a tooth which remineralizes is more resistant to caries than its own adjacent normal enamel; in my point of view, this discussion on remineralization is much more important from a cosmetics rather than a health standpoint. Bottom line, it has supported the sales of remineralizing products.

Ricardo Teles: If we have to conclude regarding the use of these supporting chemicals in mechanical plaque control in relation to periodontal disease, the message I intended to deliver is that the use of these products does not make sense as a population-based approach, as a preventive approach, at least until their cost falls considerably. For example, if we were to include Triclosan in toothpaste to be supplied to the Brazilian population, together with or through the Brazilian Ministry of Health, this product could be of some assistance in the control of gingivitis. The problem is that its cost is still too high. But it is important to bear in mind that these supporting agents have an antigingivitis effect, and that, unfortunately, some of our patients will not respond to oral hygiene or mechanical control instructions. Sometimes we get very frustrated at our office, thinking that we have failed because our patients are not flossing, or the patient has failed because he or she is not flossing. A difficult barrier to overcome arises from there, because you stop helping your patient, that is, either you do what I want or, then, "good luck". Providing options or alternatives is something quite important in this context of a more individualized service, in which there is a possibility of risk analysis, of longitudinal follow-up, and these are considerations I would to like to draw. While on the subject of white spot in relation to caries process, I would like to make an analogy with periodontal pocket. Periodontal pocket is not a periodontal disease; periodontal pocket is a lesion resulting from the periodontal disease. What means that setting the periodontal pocket as the ultimate treatment goal is something that, in my point of view, leads to certain deviations in terms of how we should treat the disease. As treating caries lesions will not control a disease process, eliminating pockets will not control a periodontal disease process. The presence of pockets serves as a risk indicator, especially pockets that remain after an initial attempt in a well-performed therapy, in which that pocket, or its maintenance, means that the patients does not have the disease process under control yet. Eliminating pockets will not solve the problem, because, to date, it has never solved it. I do not mean that, I am not making a radical apology against surgical interventions; that is not the case. But the mere interpretation that patients with residual pockets are still in need of treatment is not correct.

Mark Wolff: It is important that dentists can make use of all these preventive tools, and these tools available for patients in special risk, because restorative dentistry does not resolve these problems. Performing a restoration has never reduced the chances that a patient will continue to have the next cavity. When we think about this, we also have to examine those individuals that seem to be at risk, but are not. For example: those who, for 20 years, have had a lot of plaque, have eaten everything they should have not in terms of a healthy diet, and this person is now 20 years old, with all these risk factors, but has not developed the disease, spending public money on this person does not sound like a smart thing to do. And an individual at 45 or 50 years of age, who had never had a cavity, suddenly shows up with three carious cavities, and this is related to the fact that he is on antihypertensive, or antiasthmatic, in fact, this should be taken into account, because something has changed in the patient's life and must be well managed. Actually, we should examine these patients considering all these risk factors and drive 
our preventive efforts toward providing them with the best conditions to change this situation, because the restorative treatment alone can start a cycle, but will not resolve the problem. Thank you.

Mônica Serra: We talked about dental erosion. I would like to make it clear that dental erosion is a type of wear, and as other types of wear in general, it is associated with other factors. Dental wear may occur due to the mere presence of acid in contact with the tooth. But the fact that it can happen is related to risk, and this wear will be as great as the number of factors associated. Among associated factors, we usually find abrasion mechanical forces and different types of incident forces. In addition, it is important to understand that erosion lesions are also caused by multiple factors; there is a multicausality of these lesions. There is little evidence regarding effective treatments in the control of erosion lesions. Rinse with sodium bicarbonate solution is not as effective as expected, but, even though, we have to recommend something to our patients at times. So, this mouthrinsing could be recommended, especially following vomiting or other erosion challenge - a more efficient alternative to toothbrushing, which could associate abrasive force.

The choice of dentifrice is important in the control of abrasive-erosive wear. There is an expectation that dentifrice containing $5,000 \mathrm{ppm} F$ can assist in the control of corrosion lesions, but this has not occurred in vitro. Regarding fluoride mouthrinses, a study in vitro showed that daily use of $0.5 \%$ rather than $0.05 \%$ fluoride was effective in the control of erosion - but this has not been clinically confirmed though. So, I believe that current emphasis on treatment, as it should be in caries disease, should focus on causal factors, and pointing out, once again, that these recommendations do not fit all patients. Many people have acid food and drinks and, even though, they might not develop erosion lesions. I believe that an important and easy factor to be employed in our office is the measurement of salivary flow, because, in addition to everything we know about saliva, it plays an important role as a lubricant, reducing wear processes. So, approaching clinics, I would recommend measurement of salivary flow, evaluation of erosion risk by diet-diary analysis and recommendations mainly for those with identified risks or those patients who had lesions identified as early as possible.

Cassiano Rösing: Regarding dentin sensitivity, we are walking at a pace not as quick as we would like to, because placebo effect is too present, but we, professionals, should take some advantage of being aware of the presence of this effect to, somehow, change patients' understanding about the question of chronic pain. In addition, I would like to remind you that the smoothest strategies available for dentin sensitivity are linked to antisensitivity dentifrice, especially those containing potassium salts and fluoride (mainly high-concentrated fluoride). Besides, it is worth mentioning that questions posed by the industry, to dentists, are worthy of attention in terms of what we have discussed here during these two and a half days. Because the whole industry is stating that, there are four steps to oral hygiene, as follows: toothbrushing, flossing, dentifrice, and mouthrinsing. I believe that we understand here we do not need all four steps. Each one of us can exclude the step considered not appropriate, I am not the one to say which one is right or wrong. But assuring that everyone needs all four steps for good oral hygiene is definitely a statement based on marketing rather than evidence.

Flávio Demarco: Most of recommendations I could talk about with you are those recommendations I have already mentioned, and we hope that in a near future these recommendations are published. I think that this is really important to our dental community. We cannot get swayed by marketing strategies, we need to be very aware of our scientific evidence, but we also need to evaluate the quality of such evidence. And, today, we have very low-quality evidence available for self-care products concerning bleaching, for most of the available products. 